\title{
FRACTAL DIMENSIONS FOR UNSATURATED POROUS MEDIA
}

\author{
BOMING YU \\ Department of Physics and the State Key Laboratory of Plastic Forming and Die 8 Mold Tech. \\ Huazhong University of Science and Technology \\ 1037 Luoyu Road, Wuhan, 430074, China \\ The State Key Laboratory of Nonlinear Mechanics (LNM), Institute of Mechanics \\ Chinese Academy of Science, Beijing, 100080, China \\ yu3838@public.wh.hb.cn \\ JIANHUA LI \\ Research Center of Biomedical Materials Engineering \\ Wuhan University of Technology, Wuhan, 430070, China
}

Received October 25, 2003

Accepted October 30, 2003

\begin{abstract}
The analytical expressions of the fractal dimensions for wetting and non-wetting phases for unsaturated porous media are derived and are found to be a function of porosity, maximum and minimum pore sizes as well as saturation. There is no empirical constant in the proposed fractal dimensions. It is also found that the fractal dimensions increase with porosity of a medium and are meaningful only in a certain range of saturation $S_{w}$, i.e. $S_{w}>S_{\min }$ for wetting phase and $S_{w}<S_{\max }$ for non-wetting phase at a given porosity, based on real porous media for requirements from both fractal theory and experimental observations. The present analysis of the fractal dimensions is verified to be consistent with the existing experimental observations and it makes possible to analyze the transport properties such as permeability, thermal dispersion in unsaturated porous media by fractal theory and technique.
\end{abstract}

Keywords: Fractal; Fractal Dimension; Porous Media. 


\section{INTRODUCTION}

Katz and Thompson ${ }^{1}$ presented experimental evidence indicating that the pore spaces of a set of sandstone samples are fractals and are self-similar over three to four orders of magnitude in length extending from $10 \mathrm{~A}^{\circ}$ to $100 \mu \mathrm{m}$. They argued that the pore volume is a fractal with the same fractal dimension as the pore-rock interface. This conclusion was supported by correctly predicting the porosity from the fractal dimension, which was measured by a $\log$-log plot of number of pores versus the pore size. The correlation from their measurements is

$$
\phi=C\left(l_{1} / l_{2}\right)^{3-D_{f}}
$$

where $\phi$ is the porosity of porous sandstone, $D_{f}(=$ $2 \sim 3)$ is the fractal dimension of pores, $C$ is a constant of order one, and $l_{1}$ and $l_{2}$ are the lower and upper limits of self-similar regions, respectively.

$\mathrm{Yu}$ and $\mathrm{Li}^{2}$ derived a relation for fractal dimension for saturated/single phase porous media. This relation analytically relates the fractal dimension $D_{f}$ to porosity and microstructure parameters, $\lambda_{\max }$ and $\lambda_{\min }$, and the relation is given by

$$
D_{f}=d-\frac{\ln \phi}{\ln \frac{\lambda_{\min }}{\lambda_{\max }}}
$$

where $d$ is the Euclidean dimension, and $d=2$ and 3 in the two- and three-dimensional spaces, respectively. When $d=3$, Eq. (2) is identical with Eq. (1) as $C=1$ in Eq. (1). Equation (2) is valid not only for exactly self-similar fractal geometries such as Sierpinski carpets but also for statistically self-similar fractals such as random/disordered saturated porous media, rough surfaces and aggregates, etc. It, however, should be noted that for random/disordered porous media, $\lambda_{\max }$ and $\lambda_{\min }$ are the maximum and minimum pore sizes in a unit cell (representative cell). This also implies that the statistical self-similarity exists in the range of $\lambda_{\min } \sim \lambda_{\max }$. For the exactly self-similar fractal geometries such as Sierpinski carpets and Sierpinski gaskets, $\lambda_{\max }$ is the side length of such structures and $\lambda_{\min }=1$. Equation (2) has been successfully used to describe the fractal dimension $D_{f}$ in fractal transport models such as permeability ${ }^{3,4}$ and nucleate pool boiling heat transfer. ${ }^{5}$

Compared with the single phase (or saturated) transport phenomena in porous media, the multiphase (or unsaturated) immiscible flows (or transport) in porous media are not well understood yet. The multiphase immiscible flows in porous media are very important in practical applications such as petroleum industry, chemical engineering and soil engineering, etc. The saturated porous medium is, in fact, only the special case of the unsaturated porous medium. It is, therefore, more meaningful for practical applications to develop an analytical solution for transport properties of unsaturated (or multiphase) porous media. The fractal theory and technique may have the super potentials for such solution, and the determination of fractal dimensions of unsaturated (or multiphase) porous media may be the first step toward the solution. In this work we, therefore, focus our attention on derivation of the fractal dimensions for unsaturated porous media.

\section{FRACTAL DIMENSIONS FOR UNSATURATED POROUS MEDIA}

A porous medium consists of numerous irregular pores and solid particles (such as sandstones). According to Katz and Thompson's ${ }^{1}$ experiments, the pore volume is a fractal with the same fractal dimension as the pore-rock interface. Therefore, in this work, we only consider the pore volume fractal dimension.

Figures 1(a) and (b) show the Sierpinski carpets of stage $n=0$ and stage $n=1$, respectively. They can be considered as the idealized single phase/saturated fractal porous media, in which only a fluid (or gas) and solid are involved. In this situation, the pore area fractal dimension is described by Eq. (2) with $d=2, \lambda_{\max }=3$ and $\lambda_{\min }=1$ for Fig. 1(a), and $\lambda_{\max }=9$ and $\lambda_{\min }=1$ for Fig. 1(b).

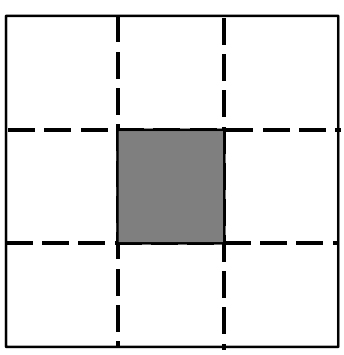

(a)

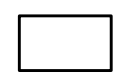

gas or non-wetting phase

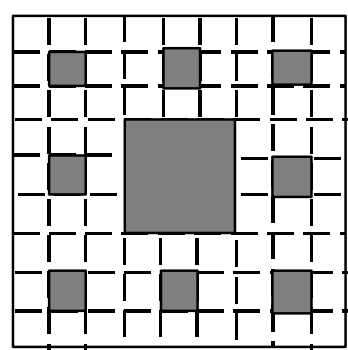

(b)

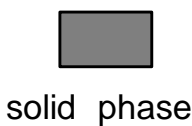

Fig. 1 Sierpinski carpets of (a) stage $n=0$, and (b) stage $n=1$. 


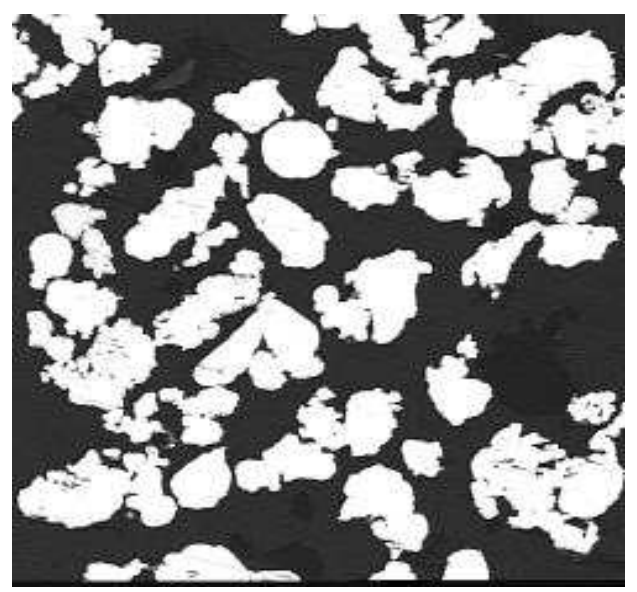

(a)

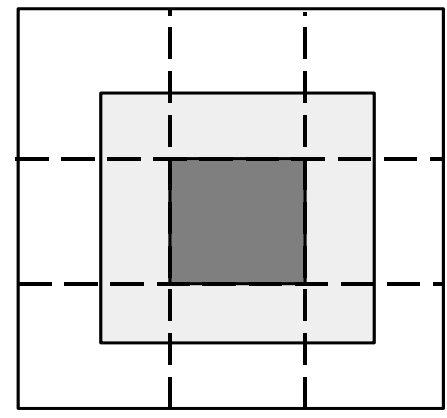

(b)

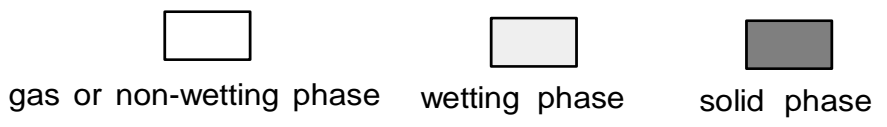

Fig. 2 (a) An image photo ${ }^{3}$ of the bi-dispersed porous medium $(\phi=0.54)$ at magnification of 50 , the white are solid and the black are pores (since the micro-pores inside clusters are very small and the cooper particles are soft, it is difficult to see the micro-pores inside clusters after the sample is polished), and (b) a unit cell for an unsaturated porous medium.

Figure 2(a) shows a real porous medium, a bi-dispersed porous medium with porosity $0.54 .^{3}$ This medium is composed of clusters (at macrolevel), which are agglomerated by small particles (at micro-level). There are macro- and micropores between and within the clusters respectively. Since the clusters and particles within the clusters are randomly distributed, the macro- and micro-pores are also randomly distributed. Figure $2(\mathrm{~b})$ is a unit cell for such a porous medium in the multi-phase/unsaturated state, in which two fluids (wetting and non-wetting) and solid are concerned, i.e. the pore is partially filled with liquid (wetting phase) and gas (non-wetting phase).
It is known that the measure of a fractal object, $M(L)$, is related to the length scale, $L$, through a scaling law in the form of 6,7

$$
M(L) \sim L^{D_{f}}
$$

where $M$ can be the length of a line, the area of a surface, the volume of a cube, or the mass of an aggregate, and $D_{f}$ is the fractal dimension of the object. Equation (3) implies the property of selfsimilarity, which means that the value of $D_{f}$ from Eq. (3) remains constant over a range of length scale, $L$.

According to Eq. (3) and Fig. 2(b), the wetting phase volume $V_{w}$ can be expressed as

$$
V_{w}=L^{D_{f, w}}
$$


where $D_{f, w}$ is the fractal dimension for wetting phase (e.g. water). In Eq. (4), the length scale $L$ has the same meaning as that in Eq. (3). For simplicity, $L$ and $V_{w}$ are taken to be dimensionless quantities, and $L=\lambda_{\max } / \lambda_{\min } \cdot{ }^{2}$ Here $\lambda_{\max }$ and $\lambda_{\min }$ have the same meanings as those defined in Eq. (2). For example, for a Sierpinski carpet of stage $n=0$, $L=3 / 1=3$; for a Sierpinski carpet of stage $n=1$, $L=9 / 1=9$.

Similarly, the non-wetting phase (or gas) volume is given by

$$
V_{g}=L^{D_{f, g}}
$$

where $D_{f, g}$ is the fractal dimension for non-wetting phase (or gas).

The total pore volume $V_{p}$ can be written as

$$
V_{p}=L^{D_{f}} \text {. }
$$

The total volume $V_{t}$ of the unit cell is

$$
V_{t}=L^{d}
$$

where $d$ is the Euclidean dimension, and $d=2$ and 3 in the two- and three-dimensional spaces, respectively.

It is clear that we have

$$
V_{w}+V_{g}=V_{p}
$$

Divided by $V_{t}$, Eq. (8) becomes

$$
\frac{V_{w}}{V_{t}}+\frac{V_{g}}{V_{t}}=\frac{V_{p}}{V_{t}}=\phi
$$

where $\phi$ is the porosity. Equation (9) can be rewritten as

$$
\phi=\phi_{w}+\phi_{g}
$$

where $\phi_{w}$ and $\phi_{g}$ are the volume fractions of wetting and non-wetting phases, respectively, and

$$
\begin{gathered}
\phi_{w}=V_{w} / V_{t} \\
\phi_{g}=V_{g} / V_{t}
\end{gathered}
$$

Due to Eqs. (4)-(7), Eq. (9) can be expressed as

$$
L^{D_{f, w}-d}+L^{D_{f, g}-d}=L^{D_{f}-d} .
$$

It is seen that Eq. (12) relates the pore volume fractal dimension $D_{f}$ to the fractal dimensions, $D_{f, w}$ and $D_{f, g}$, as well as the length scale $L$.

According to the definition ${ }^{8}$ of saturation for porous media, the saturation is given by

$$
S_{w}=V_{w} / V_{p} .
$$

For the non-wetting phase, its content $S_{g}$ is

$$
S_{g}=V_{g} / V_{p}
$$

and clearly the following relation holds

$$
S_{w}+S_{g}=1 .
$$

Due to Eqs. (9) and (13)-(15), Eq. (11) can be rewritten as

$$
\begin{gathered}
\phi_{w}=V_{w} / V_{t}=V_{w} \phi / V_{p}=S_{w} \phi \\
\phi_{g}=V_{g} / V_{t}=V_{g} \phi / V_{p}=S_{g} \phi=\left(1-S_{w}\right) \phi .
\end{gathered}
$$

From Eqs. (4)-(7), Eq. (11) can be also expressed as

$$
\begin{gathered}
\phi_{w}=\frac{V_{w}}{V_{t}}=\frac{L^{D_{f, w}}}{L^{d}}=L^{D_{f, w}-d} \\
\phi_{g}=\frac{V_{g}}{V_{t}}=\frac{L^{D_{f, g}}}{L^{d}}=L^{D_{f, g}-d} .
\end{gathered}
$$

Combining Eqs. (16) and (17) yields

$$
\begin{gathered}
D_{f, w}=d+\frac{\ln \left(S_{w} \phi\right)}{\ln L} \\
D_{f, g}=d+\frac{\ln \left[\left(1-S_{w}\right) \phi\right]}{\ln L}
\end{gathered}
$$

where $L=\lambda_{\max } / \lambda_{\min }$. Equation (18-1) indicates that when $S_{w}=1$, Eq. (18-1) will be reduced to Eq. (2), meaning that the medium becomes a single phase/saturated porous one. If $S_{w}=0$, Eq. (18-2) will be also reduced to Eq. (2) and the medium is also a single phase/saturated porous one. It can be seen that Eq. (2) is only a special case of saturation $S_{w}=1$ in Eq. (18-1) or $S_{w}=0$ in Eq. (18-2), and Eq. (18) is the general expression for fractal dimension for porous media, including both saturated and unsaturated porous media.

\section{RESULTS AND DISCUSSIONS}

To illustrate the determination of the fractal dimensions, we take the bi-dispersed porous medium [see Fig. 2(a)] as an example of a real porous medium. Consider the medium is in the unsaturated or in the three-phase state at porosity 0.54 .

In order to determine the fractal dimensions of phases, we need to calculate the length scale $L=$ $\lambda_{\max } / \lambda_{\min }$ in Eq. (18). The maximum pore size for the bi-dispersed porous media is given by ${ }^{3}$

$$
\lambda_{\max }=R \sqrt{2 \frac{\phi-\phi_{i}}{1-\phi}}
$$

where $R$ is the characteristic cluster radius, and $\phi_{i}$ is the porosity inside the cluster and given by ${ }^{9}$

$$
\phi_{i}=0.347 \phi \text {. }
$$




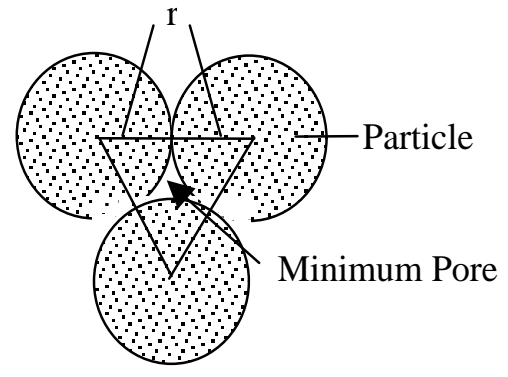

Fig. 3 The possible arrangement of particles inside a cluster forms a minimum pore.

The minimum pore size $\lambda_{\min }$ can be determined by the possible arrangement (see Fig. 3) of particles touching each other inside a cluster,

$$
\lambda_{\min }=\frac{2 r}{\sqrt{\pi}} \sqrt{\sqrt{3}-\pi / 2}
$$

where $r$ is the radius of particle in a cluster. It is generally assumed that the macro- and micropore sizes have the same order of magnitude as the characteristic cluster and particle sizes, respectively. Thus, we take $R / r=400 / 80 .^{9}$ Therefore, we obtain the ratio of

$$
L=\frac{\lambda_{\max }}{\lambda_{\min }}=\frac{5 \sqrt{2}}{2} \frac{\sqrt{\pi\left(\phi-\phi_{i}\right) /(1-\phi)}}{\sqrt{\sqrt{3}-\pi / 2}} .
$$

It is seen that $L$ depends on porosity only, independent of saturation.

Inserting Eq. (22) into Eq. (18) with $d=2$, we can calculate the fractal dimensions $D_{f, w}$ and $D_{f, g}$ under different saturations and porosities. Figure 4 presents the fractal dimensions, $D_{f, w}$ and $D_{f, g}$, versus saturation $S_{w}$ at different porosities in two dimensions $[d=2$ in Eq. (18)]. It is seen from Fig. 4 that the fractal dimension $D_{f, w}$ increases monotonously with saturation. As saturation tends to 1 , the fractal dimension $D_{f, w}$ reaches its maximum possible value of about 1.78 [see Fig. $4(\mathrm{a})$ ] at porosity 0.54 , approximately the same value as that of 1.81 measured by the box-counting method $^{3}$ for the medium at porosity 0.54 . This is expected because when saturation tends to 1 , the unsaturated porous medium is close to a single phase/saturated porous medium, Eq. (18-1) gives the result of $D_{f, w}=1.78$, very close to the result of 1.81 measured by the box-counting method ${ }^{3}$ for the real bi-dispersed porous medium at porosity 0.54 . The similar phenomenon can be observed for the non-wetting phase. The fractal dimension $D_{f, g}$ reaches its maximum possible value 1.78 [see Fig. 4(b)] as saturation is close to zero at porosity 0.54 . This means that as saturation tends to zero, the medium is fully filled with a non-wetting fluid (or single-phase fluid), so it is expected that the fractal dimension is exactly the same as that for the saturated porous medium. Figure 4 also shows that the fractal dimensions, both $D_{f, w}$ and $D_{f, g}$, depend on porosity. The higher the porosity, the higher the fractal dimension. This can be interpreted that the higher porosity implies larger pore area, the larger pore area leads to the higher phase volume and the higher fractal dimension. In the limiting case, as porosity tends to 1 , a unit cell of the medium becomes a smooth plane, whose fractal dimension is 2. Therefore, the present results are reasonable. From Fig. 4, an important phenomenon can be also found. That is when saturation $S_{w}<S_{\min }(=0.1$,

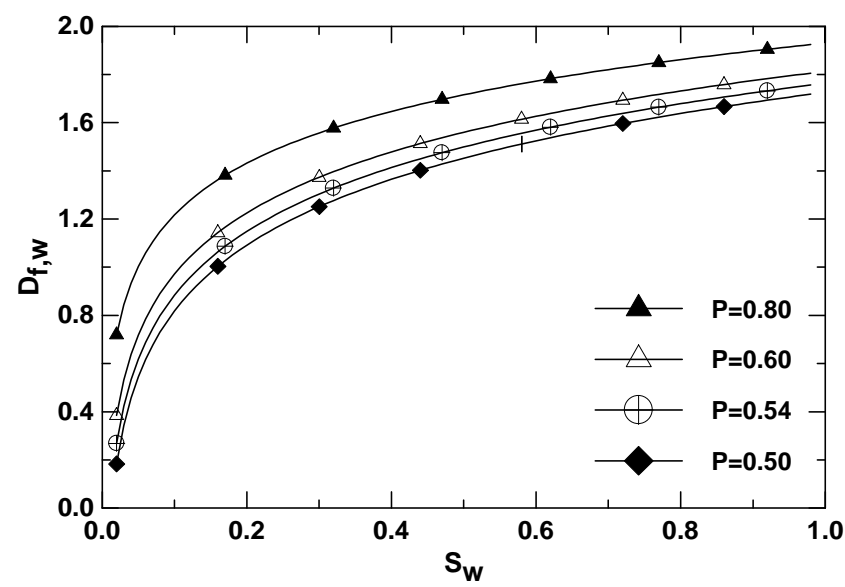

(a)

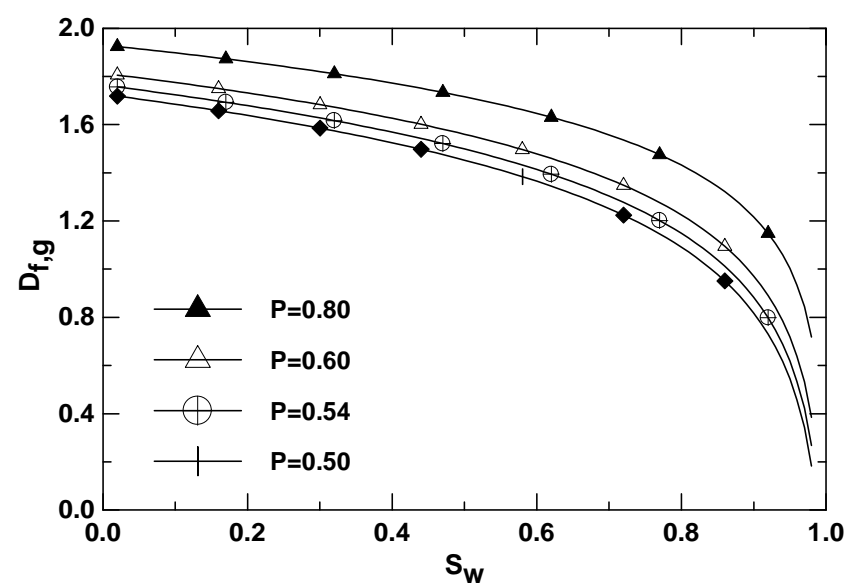

(b)

Fig. 4 The phase fractal dimensions, $D_{f, w}$ and $D_{f, g}$, versus saturation at different porosities, P. 
at $\phi=0.60)$, the fractal dimension $D_{f, w}<1$. This reveals that when saturation $S_{w}<S_{\min }(=0.1$, at $\phi=0.60)$, the wetting phase distribution in porous media is non-fractal according to fractal theory. The value of $S_{\min }$ is, thus, defined as the lower limit of saturation, below which the wetting phase becomes non-fractal. From Fig. 4(a), it is also seen that the lower limit of saturation is also related to porosity. At lower porosity such as at $\phi=0.50$, the lower limit of saturation is about 0.2. Similarly, when saturation $S_{w}>S_{\max }(=0.9$, at $\phi=0.60), D_{f, g}<1$. This means that the non-wetting phase distribution is also non-fractal when $S_{w}>S_{\max }(=0.9$, at $\phi=0.60)$. The value of $S_{\max }$ is, therefore, defined as the upper limit of saturation, above which the nonwetting phase becomes non-fractal. This suggests that only when $S_{w}>S_{\min }$ and $S_{w}<S_{\max }$ at a given porosity, the wetting and non-wetting phases are fractal objects, respectively. On the other hand, according to literature ${ }^{10}$ by Kaviany, at very low saturation the wetting phase becomes disconnected (or immobile), and at very high saturation the nonwetting phase becomes disconnected (or immobile). Usually, the experimentally relative permeability data $^{8,10}$ were reported also in the ranges of about $S_{w}>0.1 \sim 0.2\left(=S_{\text {min }}\right)$ for wetting phase and about $S_{w}<0.9\left(=S_{\max }\right)$ for non-wetting phase. Thus, the present analysis is consistent with the experimental observations. The fractal dimensions, $D_{f, w}$ and $D_{f, g}$, are meaningful only in the ranges of $S_{w}>S_{\min }$ for wetting phase and $S_{w}<S_{\max }$ at a given porosity for non-wetting phase for the present bi-dispersed porous medium for requirements from both fractal theory and experimental observations.

\section{CONCLUDING REMARKS}

A complete analysis of fractal dimension for both saturated and unsaturated porous media is presented in this paper. The general expressions for the fractal dimensions, $D_{f, w}$ and $D_{f, g}$, given by Eqs. (18-1) and (18-2), are expressed in terms of porosity $\phi$, saturation $S_{w}$ and microstructure parameters, $\lambda_{\max } / \lambda_{\min }$. There is no empirical constant in the proposed fractal dimensions. The fractal dimension Eq. (2) is only a special case of the unsaturated porous medium by setting $S_{w}=1$ in
Eq. (18-1) or by setting $S_{w}=0$ in Eq. (18-2). The fractal dimensions, $D_{f, w}$ and $D_{f, g}$, are meaningful only in the ranges of $S_{w}>S_{\min }$ for wetting phase and $S_{w}<S_{\max }$ for non-wetting phase at a given porosity for the present real bi-dispersed porous medium for requirements from both fractal theory and experimental observations. The present analysis of the fractal dimensions is verified to be consistent with the experimental observations and it makes possible to analyze the transport properties such as permeability, thermal dispersion in unsaturated porous media by fractal theory and technique. This work is currently in process.

\section{ACKNOWLEDGMENTS}

This work was supported by the National Natural Science Foundation of China through grant No. 10272052.

\section{REFERENCES}

1. A. J. Katz and A. H Thompson, "Fractal Sandstone Pores: Implications for Conductivity and Pore Formation," Phys. Rev. Lett. 54, 1325-1328 (1985).

2. B. M. Yu and J. H. Li, "Some Fractal Characters of Porous Media," Fractals 9, 365-372 (2001).

3. B. M. Yu and P. Cheng, "A Fractal Model for Permeability of Bi-dispersed Porous Media," Int. J. Heat Mass Transfer 45, 2983-2993 (2002).

4. B. M. Yu, J. H. Li and D. M. Zhang, "A Fractal Trans-plane Permeability Model for Textile Fabrics," Int. Commun. Heat Mass Transfer 30, 127-138 (2003).

5. B. M. Yu and P. Cheng, "A Fractal Model for Nucleate Pool Boiling Heat Transfer," J. Heat Transfer 124, 1117-1124 (2002).

6. B. B. Mandelbrot, The Fractal Geometry of Nature (W. H. Freeman, San Francisco, 1982).

7. J. Feder, Fractals (Plenum Press, New York, 1988).

8. J. Bear, Dynamics of Fluids in Porous Media (American Elsevier Publishing Company Inc., New York, 1972).

9. Z. Q. Chen, P. Cheng and C. H. Hsu, "A Theoretical and Experimental Study on Stagnant Thermal Conductivity of Bi-dispersed Porous Media," Int. Commun. Heat Mass Transfer 27, 601-610 (2000).

10. M. Kaviany, Principles of Heat Transfer in Porous Media, 2nd ed. (Springer-Verlag, New York, Inc., 1995). 